\title{
Du panafricanisme à la réafricanisation : formation et actualité du mouvement akan aux États-Unis
}

Pauline Guedj

\section{CpenEdition}

1 Journals

Édition électronique

URL : https://journals.openedition.org/jsa/2832

DOI : 10.4000/jsa.2832

ISSN : 1957-7842

Éditeur

Société des américanistes

Édition imprimée

Date de publication : 5 janvier 2005

Pagination : 93-112

ISSN : 0037-9174

\section{Référence électronique}

Pauline Guedj, « Du panafricanisme à la réafricanisation : formation et actualité du mouvement akan aux États-Unis », Journal de la Société des américanistes [En ligne], 91-1 | 2005, mis en ligne le 10 janvier 2010, consulté le 02 septembre 2022. URL : http://journals.openedition.org/jsa/2832 ; DOI https://doi.org/10.4000/jsa.2832 


\title{
DU PANAFRICANISME À LA RÉAFRICANISATION : FORMATION ET ACTUALITÉ DU MOUVEMENT AKAN AUX ÉTATS-UNIS
}

\author{
Pauline GUEDJ *
}

\begin{abstract}
À Cuba et au Brésil, la réafricanisation des religions afro-américaines est centrée autour de la valorisation des origines yoruba de ces cultes. En se fondant sur une enquête de terrain réalisée aux États-Unis, cet article montre comment l'originalité de la réafricanisation en Amérique du Nord réside dans l'existence, à côté de la religion des orisha, d'un autre système de croyance "africain » : celui promu par le mouvement akan. Entretenant entre eux d'étroites relations, les deux systèmes yoruba et akan donneront alors naissance à un nouveau syncrétisme que nous qualifierons de panafricain. [Mots clés : États-Unis, Afro-Américains, réafricanisation, syncrétisme, panafricanisme, akan, yoruba.]
\end{abstract}

From panafricanism to reafricanizacion : Formation and realities of the Akan movement in the United States. In Cuba and in Brazil, the reafricanization of African American religions has been centered on the promotion of their Yoruba origins. Based on a fieldwork conducted in the United States in these past two years, this article shows how the specificities of North America in terms of reafricanization lie in the existence in this country of another "African " religion : the Akan religion. Always in close connections in North America, the Yoruba and the Akan movements will then give birth to a new syncretism that the author refers to as " Pan-African ». [Key words : United States of America, African Americans, reafricanization, syncretism, panafricanism, Akan, Yoruba.]

Del panafricanismo a la reafricanización : formación y actualidad del movimiento akan en los Estados Unidos. En Cuba como en Brasil, la idea de un proceso de reafricanización de las religiones afro-americanas gira en torno a la valorización del origen yoruba de estos cultos. Con base en la experiencia de un trabajo de campo realizado en los Estados Unidos, este artículo muestra de qué manera la originalidad de la reafricanización en América del Norte reside no sólo en la existencia de la religión de los Orichas, sino también en la presencia de otro sistema de creencias « africano » difundido por el movi-

* Laboratoire d'ethnologie et de sociologie comparative, UMR 7535, Université de Paris XNanterre [GuedjPP@aol.com].

Journal de la Société des Américanistes, 2005, 91-1, pp. 93-112. OS Société des Américanistes. 
miento akan. Estableciendo relaciones estrechas, los dos sistemas yoruba y akan dan nacimiento a un nuevo sincretismo que el autor califica de panafricano. [Palabras claves: Estados Unidos, afro-americanos, reafricanización, sincretismo, panafricanismo, akan, yoruba.]

Aujourd'hui, au Brésil, à Cuba, aux États-Unis ou en Argentine, des religions afro-américaines comme le candomblé ou la santería sont l'objet d'une tentative de réafricanisation de leurs pratiques et croyances. Au Brésil ${ }^{1}$, certains terreiros, ou maisons de culte, dits traditionnels cherchent à « purifier » le candomblé de ses influences catholiques et spirites, en revendiquant l'utilisation de la langue yoruba dans le rituel ou en y introduisant la divination par les odìs ${ }^{2}$ jusqu'alors absente du champ religieux afro-brésilien. À Cuba ${ }^{3}$, où un phénomène comparable est observable, c'est cette même divination par le système d'ifà qui sera érigée au statut de pratique spirituelle la plus pure et authentiquement africaine dans l'île et par conséquent la plus valorisée. Il s'agira alors, pour les tenants de cette réafricanisation, de " désyncrétiser » la santería ou le candomblé, d'ôter le masque ${ }^{4}$ derrière lequel leurs pratiquants avaient dissimulé les divinités africaines pendant des années de domination servile afin de retrouver aujourd'hui des pratiques jugées originelles, intactes, essentiellement africaines et authentiques.

Réafricanisés, la santería et le candomblé vont ainsi devenir des éléments constitutifs d'un vaste système de croyances centré autour de la vénération des orisha, divinités d'origine yoruba et présentes dans chacune de ces pratiques religieuses. Remodelés artificiellement selon un modèle "yoruba », que des décennies de recherches anthropologiques ont dangereusement érigé au statut de tradition africaine la plus noble et «civilisée » ${ }^{5}$, la santería et le candomblé deviendront des variantes locales d'une religion globale, la religion des orisha. Religion partagée par des croyants originaires de l'Afrique comme du Nouveau Monde, celle-ci se constituera par une série de réseaux créés entre l'Afrique et plusieurs foyers américains dont certains, comme Salvador de Bahia, seront qualifiés de traditionnels. Rassemblés tous les ans dans des congrès itinérants appelés COMTOC (Conférences mondiales sur la tradition et la culture des orisha), certains membres de cette nouvelle communauté de croyants vont chercher à établir leur dogme et leurs pratiques et à revendiquer haut et fort leur position d'adeptes d'une religion mondiale ou World Religion.

Aux États-Unis ${ }^{6}$, l'expérience de réafricanisation de la communauté afroaméricaine est née, à la fin des années 1950 , de la rencontre des théories du nationalisme culturel ${ }^{7}$ et des religions afro-américaines introduites sur le territoire grâce à l'immigration massive des populations haïtiennes, portoricaines et surtout cubaines. Les années 1950-1960 marquèrent un important tournant dans l'histoire des Noirs américains. Alors que la ségrégation légale avait été abolie en 1964 grâce aux efforts de Martin Luther King et du Civil Rights Movement, que l'Afrique, de l'Ouest en particulier, entrait dans un long processus de décolonisa- 
tion et que des phénomènes de lutte contre le racisme et l'oppression étaient également observables dans les Caraïbes et en Amérique du Sud, les Noirs américains prirent conscience de leur habilité à transformer le système en place. Les années 1960 marquèrent, ainsi, le début de ce que l'on appela le Black Power, scène d'une véritable explosion des revendications noires et des premiers aflichages répétés de la fierté raciale au sein de la communauté afro-américaine.

Parmi les différentes tendances du Black Power, le nationalisme culturel constitua une orientation politique influente. S'opposant aux revendications révolutionnaires du Black Panther Party et d'autres organisations d'inspiration marxiste de ce type, les nationalistes culturels, de Amiri Baraka à Maulana Karenga, prônèrent le retour des Afro-Américains à ce qui était considéré comme leur culture ancestrale, une culture qui, selon ces deux leaders, façonnait et conditionnait leur être. Pour les tenants du nationalisme culturel, les AfroAméricains devaient, en effet, s'en remettre à une Afrique largement mystifiée pour s'en inspirer dans leur quotidien et se séparer ainsi " mentalement » et "psychologiquement » du monde blanc. Cherchant à refaire corps avec leur culture et héritage africains pour pouvoir revendiquer une identité propre aux États-Unis qui n'ait pas souffert des mutilations causées par l'esclavage et qui n'ait pas été conditionnée par les valeurs de la société américaine, les nationalistes culturels vont trouver dans la santería cubaine une voie privilégiée pour partir à la quête de leurs origines. Forts de cette découverte, les Noirs américains vont alors intégrer les maisons de culte cubaines et tenter de s'y faire initier.

Toutefois, la cohabitation entre les Cubains et les Afro-Américains dans des maisons de culte communes va s'avérer de plus en plus conflictuelle. Alors que les Afro-Américains critiquent les influences catholiques présentes dans la santería et principalement le culte des saints qui la caractérise, les Cubains contestent les opérations mises en œuvre par certains activistes noirs américains qui cherchent à élaborer, à travers la pratique de la santería, une politique de libération du peuple noir. Aux États-Unis, la réafricanisation est donc, dans un premier temps, le résultat d'une réaction des Afro-Américains à la santería cubaine. Désirant se séparer des Cubains, ceux-ci vont fonder leurs propres maisons de culte qualifiées alors de yoruba, se revendiquer des liens ancestraux avec les Yoruba du Nigeria et reproduire certaines pratiques qu'ils jugent comme authentiquement africaines, telle la polygamie.

Mais, à côté du mouvement yoruba, s'est développé aux États-Unis un second mouvement qui, lui aussi, va jouer un rôle majeur, à la fois dans la constitution d'un champ religieux « africain » en Amérique du Nord et dans l'élaboration des actuels discours nationalistes afro-américains. Fondé, lui aussi, entre la fin des années 1950 et la première moitié des années 1960, ce mouvement s'appuiera sur la formation de réseaux créés, non pas avec les « Yoruba de la diaspora » ${ }^{8}$ et du Nigeria, mais avec les Akan du Ghana. Prônant la vénération de divinités appelées abosom ${ }^{9}$, les pratiquants américains de la religion akan vont tenter de se 
rassembler avec leurs « homologues » africains pour créer une communauté de croyants transnationale ${ }^{10}$ se déployant au côté de celle formée par les fidèles de la religion des orisha.

En revenant sur la formation et l'évolution du mouvement akan aux ÉtatsUnis, cet article cherchera à montrer comment la réafricanisation en Amérique du Nord trouvera son originalité dans la coexistence en son sein de deux systèmes de croyance distincts, conçus tous deux comme purement africains et qui entretiennent entre eux d'étroites relations. Si la réafricanisation au Brésil ou à Cuba était prônée principalement sous la bannière des Yoruba du Nigeria, la présence aux États-Unis d'un mouvement akan témoigne de la prégnance d'une connexion politique et panafricaniste faisant date entre l'Afrique et la communauté noire américaine. Entre Akan et Yoruba, les adeptes de la réafricanisation aux ÉtatsUnis oscilleront sans cesse entre la revendication d'une appartenance à une " ethnie » transnationale ${ }^{11}$, dont l'origine africaine est à chercher soit au Nigeria, soit au Ghana, et le désir de raviver spirituellement une Afrique ancestrale, « re-territorialisée » aux États-Unis.

\section{LA NAISSANCE DU MOUVEMENT AKAN}

Le mouvement akan est né en 1965, lorsqu'un musicien percussionniste afro-américain du nom de Gus Dinizulu part en tournée en Afrique de l'Ouest avec sa compagnie de danse. Déjà très impliqué dans la valorisation de l'héritage africain des populations afro-américaines des États-Unis, Dinizulu profite de ce voyage pour découvrir la culture et les traditions de ses ancêtres. La tournée passera par le Nigeria et la Côte d'Ivoire, mais c'est au Ghana que le leader de la compagnie rencontrera son initiatrice dans une religion qu'il adoptera et qu'il développera en Amérique du Nord.

Au Ghana, Dinizulu séjournera dans la ville de Larteh Kubease, ville où se situe un sanctuaire très populaire dans le pays : l'Akonedi shrine. C'est là qu'il fera la rencontre de Nana Akua Oparebea, okomfohene ou prêtresse en chef du sanctuaire, qui l'accueillera et effectuera pour lui une divination cherchant à la fois à déterminer l'origine de ses ancêtres africains et la nature de sa mission au sein de la communauté afro-américaine des États-Unis. À l'issue de la séance, Oparebea révèlera à Dinizulu que ses ancêtres étaient originaires d'un village akan voisin et qu'ils étaient issus du propre clan paternel de la prêtresse. De plus, Dinizulu et Oparebea recevront, lors de cette divination, la mission divine de partir à la recherche de ces Noirs américains qui, comme Dinizulu, auraient des ancêtres akan originaires du Ghana et, plus particulièrement, de la région de l'A kwapem, chaîne montagneuse située à quelques kilomètres d'Accra, où se pratique le culte de la divinité Akonedi, principale divinité du sanctuaire. 
Dinizulu, "missionnaire » de l'Akonedi shrine en Amérique, se voit alors remis le titre d'omanhene, " chef des Akan d'Amérique », et sera rebaptisé Nana Yao Opare Dinizulu, Nana étant le titre honorifique décerné à un aîné, Yao désignant le jour de la semaine où celui-ci vint au monde, un jeudi, et Opare le nom du clan de ses ancêtres, clan paternel de la prêtresse en chef de l'Akonedi shrine. Enfin, lors de cette première visite au Ghana, Dinizulu obtiendra un premier enseignement sur le culte des abosom, divinités akan vénérées au sanctuaire, et sera nommé abosomfo, gardien des autels de ces entités spirituelles.

De retour aux États-Unis, Dinizulu cherchera immédiatement à honorer sa mission en continuant à entretenir des rapports étroits avec Nana Akua Oparebea et en se lançant dans une véritable campagne de conversion visant à raviver les origines akan de ses concitoyens afro-américains. En 1968, Dinizulu fait l'acquisition d'un local où il instaurera un centre culturel et un temple akan. Située dans le Queens de New York, à Long Island City, la structure accueille des cours de danse et de percussions, des expositions et des conférences sur l'Afrique et les Akan, ainsi que des cérémonies publiques, akom, organisées sous la coupe de Dinizulu.

De son côté, Oparebea accueillera au Ghana de nombreux Afro-Américains qui, à partir de ce premier contact, vont eux aussi chercher à redécouvrir leurs origines et, même pour certains, à se faire initier à la prêtrise akan. De plus, elle va effectuer entre 1965 et 1995 (année de son décès) neuf voyages aux États-Unis, à New York, Washington et Philadelphie principalement, grâce auxquels elle pourra contrôler le bon fonctionnement des rituels mis en œuvre par les AfroAméricains, orchestrer certaines cérémonies, envoyer d'autres prêtres ghanéens aux États-Unis et installer des autels consacrés au Ghana dans les principales maisons de culte américaines.

Le mouvement akan est donc né de la rencontre d'un nationaliste culturel afro-américain, musicien à la tête d'une compagnie de danse africaine, et de la prêtresse en chef d'un lieu de culte traditionnel au Ghana. Là où le mouvement yoruba se fondait sur la lutte contre le syncrétisme à l'intérieur d'une religion afro-cubaine, les membres du mouvement akan, souvent eux-mêmes anciens participants aux cérémonies santeras, vont chercher à s'initier à une religion qui n'a jamais souffert des abus de l'esclavage. Au lieu de s'affilier à une religion afro-américaine qu'ils tenteraient de " purifier », les "Akan-américains » vont immédiatement se forger des rapports directs avec l'Afrique, ses pratiques religieuses et ses officiants. Or l'initiative de cette série de rapprochements entre les Afro-Américains et l'Afrique, le Ghana en particulier, ne revient pas uniquement à Dinizulu et à ses compagnons de route. Le panafricanisme, idéologie énoncée et défendue depuis la fin du XIX ${ }^{\mathrm{e}}$ siècle, à la fois par de nombreux Afro-Américains et par certains Ghanéens, constituait déjà un terreau fertile qui favorisera la formation des réseaux akan entre la Terre Mère et le Nouveau Monde. 
Africa MUST UNITE : RÉSEaUX aKan ET Panafricanisue

Lorsque Dinizulu pénètre dans le sanctuaire d'Akonedi en 1965, la communauté afro-américaine des États-Unis et le Ghana partagent déjà une importante tradition de contacts et de luttes communes ${ }^{12}$. Kwame Nkrumah, premier Président de la république libre du Ghana, a effectué une partie de sa formation universitaire aux États-Unis. En effet, en 1935, celui-ci intègre la Lincoln University, une université noire de Pennsylvanie. Quelques années plus tard, il rejoint la University of Pennsylvania où il obtient en 1943 un master en philosophie. Aux États-Unis, Nkrumah découvre les écrits du prédicateur Marcus Garvey ${ }^{13}$, militant jamaïcain, qui prône la formation d'un empire économique établi entre les populations noires de l'Afrique et des Amériques, et de l'intellectuel noir américain, W. E. B. Du Bois ${ }^{14}$. Les prises de position de ce dernier exerceront une influence décisive sur l'engagement politique du futur leader ghanéen. Dans les années 1960, Nkrumah invita d'ailleurs Du Bois à séjourner à Accra, pour s'atteler tous deux à la rédaction de l'Encyclopedia Africana, vaste projet censé servir de fondement théorique à la lutte pour l'unification du continent africain ${ }^{15}$.

Dès son retour en Afrique, en 1947, Nkrumah considère donc que la lutte des Africains contre le colonialisme et l'oppression ne peut faire l'économie d'une collaboration avec ces Afro-Américains qui cherchent à se libérer du joug de la ségrégation. Bien qu'ayant eu lieu en Amérique, la lutte des esclaves pour leur émancipation et, surtout, les prises de position de W. E. B. Du Bois constituent pour Nkrumah (1968, p. 5) des « trésors de l'histoire africaine ». Ayant participé à la $V^{\mathrm{e}}$ Conférence Panafricaine organisée à Manchester en 1945, Nkrumah devient l'un des principaux défenseurs de l'unification du peuple noir comme seul remède à ses maux actuels.

Or, au même moment où se constitue un État ghanéen indépendant sous la tutelle de Kwame Nkrumah, l'Akonedi shrine, qui sera central dans la formation de réseaux akan entre le Ghana et les États-Unis, va devenir un important élément dans la valorisation d'une " traditionalité » ghanéenne, constitutive de cette « personnalité africaine » qui caractériserait les "Africains » vivant des deux côtés de l'Atlantique ${ }^{16}$.

Ainsi en 1960, Akua Oparebea guérit Mensah Dapaa, un ami de Nkrumah, professeur de botanique à l'université de Legon, à Accra. C'est lui qui, en devenant le Scientific and educational attaché du sanctuaire, utilisera l'exemple du culte d'Akonedi et de ses divinités tutélaires pour légitimer la pratique de la médecine traditionnelle dans un Ghana qui cherche à défendre ses spécificités africaines sur la scène internationale ${ }^{17}$. Cette initiative sera reprise, en 1962, par Kwame Nkrumah lui-même, qui fera de l'Akonedi shrine le quartier général d'une association nationale de guérisseurs, la Ghana psychic and traditional healing association $^{18}$, association dont Akua Oparebea prendra la tête. La Ghana psychic and traditional healing association, avec l'aide de l'État, se propose ainsi de 
reconnaître l'ensemble des techniques utilisées par les thérapeutes du pays et de lutter contre les possibles usurpateurs.

Dès les années 1960, l'Akonedi shrine est ainsi utilisé par le pouvoir ghanéen à la fois pour légitimer la pratique de la médecine traditionnelle au Ghana et pour tenter de fonder une unité nationale des thérapeutes dans une association unique. Rassemblant en son sein et sous l'appellation « akan » des divinités originaires de l'ensemble du territoire ghanéen et donc souvent elles-mêmes non akan, l'Akonedi shrine devient ainsi un emblème de la lutte pour l'unité nationale que Kwame Nkrumah mena en tentant de réduire les résistances de certains groupes ethniques réfractaires à la construction d'un État centralisé ${ }^{19}$. Lorsque les premiers Afro-Américains pénètrent dans le sanctuaire, c'est donc selon cette même logique d'unité de la Nation et de l'ensemble du peuple noir qu'Oparebea les accueille. Nkrumah, favorable à ce processus de diffusion des traditions akan aux États-Unis, intervient d'ailleurs directement auprès du conseil des aînés de l'Akonedi shrine pour les convaincre de laisser Oparebea voyager en Amérique et y introduire les divinités. Erigé par le gouvernement ghanéen en symbole d'une tradition africaine, l'Akonedi shrine va constituer, pour les Afro-Américains, un " centre de formation » qui leur apprendra à (re)devenir Africains et leur enseignera les fondements de cet "ethos» qu'ils sont censés partager avec leurs « homologues » de la Terre Mère et qui les rassemble dans une communauté déjà transnationale et panafricaine.

\section{LE MOUVENENT AKAN AUJOURD'HUI}

Aujourd'hui, le mouvement akan est constitué d'une série de maisons de culte ou shrine houses qui virent le jour au début des années 1970, sur la côte Est du pays principalement. Ces maisons de culte, placées sous la tutelle d'un aîné (nana), rassemblent des okomfowaa, personnes destinées à la prêtrise, des okomfo, prêtres ne dirigeant pas leur propre maison et, souvent, ne possédant pas de filleuls ou godchildren, enfin de simples pratiquants qui participent à certaines activités religieuses, notamment aux akom ou cérémonies publiques. La plupart des maisons de culte sont composées de la chambre du ou de la nana, d'une salle de réunion, où sont organisés des cours d'introduction aux traditions akan et à la langue twi, et d'une chambre des autels, shrine room, où sont rassemblés les autels des principales entités vénérées. Seuls les shrines dédiés à la divinité Tegare et, le cas échéant, à la divinité yoruba Eshu sont parfois disposés non pas dans cette chambre, mais à l'entrée de la maison. Quant aux autels des ancêtres du ou de la nana, ils sont installés soit dans sa chambre à coucher, soit dans une section spécifique de la shrine room.

Chaque maison de culte akan entretient des relations de filiation avec l'Akonedi shrine de Larteh Kubease et Akua Oparebea en particulier ${ }^{20}$. Il existe en fait 
quatre lignées akan, aux États-Unis, fondées pour la plupart par les premiers Afro-Américains qui firent le pèlerinage au Ghana. À New York, le lignage religieux Dinizulu constitue la lignée akan la plus ancienne. À Philadelphie, la lignée Korantemaa a été initiée par une prêtresse, danseuse de la célèbre compagnie Arthur Hall Afro-American Dance Ensemble, Nana Korantemaa Ayeboafo, qui séjourna pendant près de sept ans à Larteh Kubease, auprès d'Oparebea, et qui s'est vu remettre, il y a quelques années, la fonction d'okomfohene, prêtresse en chef des Akan d'Amérique par certains officiants de l'Akonedi shrine. À Washington, le lignage religieux Brown fut amorcé par Nana Kivabena Brown, ancien porte-parole de Dinizulu ayant fait sécession. C'est ce dernier qui accueillit en 1978 l'okomfo ghanéenne, Nana Nsia Dennis, qui à son tour initia, à New York, une quatrième lignée. Seule Ghanéenne directement impliquée dans le mouvement aux États-Unis, Nana Nsia Dennis y fut envoyée par Akua Oparebea pour qu'elle enseigne aux Afro-Américains certains rituels ou shrine works. Aujourd'hui, elle est membre de l'association The children of Akonnedi dans laquelle elle joue le rôle de spécialiste du rituel - notamment des libations qu'elle est la seule à verser en utilisant exclusivement la langue twi.

Quel que soit le lignage religieux akan auquel il appartient, le nouveau membre d'une maison de culte va devoir participer à plusieurs rituels qui certifient son lien de filiation avec l'Akonedi shrine et les Akan du Ghana, et qui révèlent au grand jour son appartenance au mouvement. En lui offrant une nouvelle identité et en le plaçant sous la protection de divinités qui l'accepteront et le reconnaîtront comme un de leurs "enfants d'Amérique ", ces rituels ont pour but de transformer le pratiquant afro-américain en représentant légitime de la tradition akan aux États-Unis.

L'ordre d'exécution des trois rituels qui précèdent la possible initiation du membre à la prêtrise varie grandement selon les personnes et les maisons de culte. En général, le nouveau sympathisant à la shrine house va rapidement, lors d'une séance de divination ou reading ${ }^{21}$, recevoir un nom akan, shrine name, composé d'une particule correspondant au jour de la semaine où il est né et d'un patronyme lié à un clan de Larteh Kubease, voire à l'appellation décernée à un officiant de l'Akonedi shrine décédé (Figure 1). Descendant, en même temps que « réincarnation » américaine d'une personnalité larthérienne, l'« Akan-américain » va revendiquer son nouveau nom comme sa seule et unique identité ${ }^{22}$ et placer des représentations de son " homologue » africain sur les autels de ses ancêtres. Le nom Abena Oparebea, par exemple, désignera une femme américaine née un mardi à la fois descendante du clan maternel d'Oparebea et représentante de la prêtresse dans le Nouveau Monde.

À côté de cette pratique du changement de nom, le ou la nana de la maison de culte va exécuter sur le bras droit de son godchild des scarifications, symbolisant sa connexion avec l'A konedi shrine de Larteh Kubease. Ligne verticale à partir de laquelle émergent des embranchements en diagonale, ces scarifications, appelées 


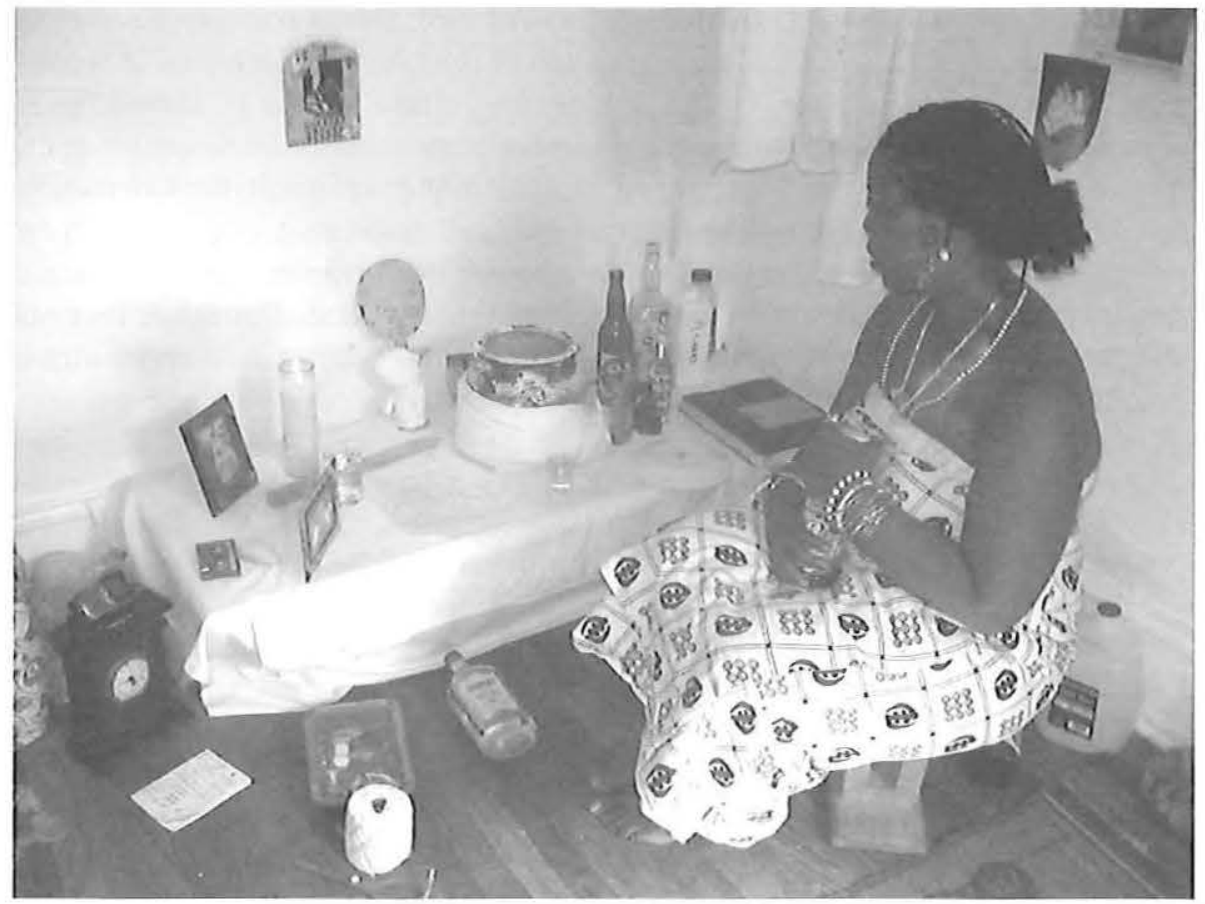

FIG. 1 - À Philadelphie, okomfo Nyo Agyiriwah effectue une divination devant l'autel de la divinité Asuo Gyebi.

tree (arbre), évoquent les liens qui existent entre l'Américain et sa maison mère larthérienne. Le tree symbolisera cet arbre généalogique qui allie "Akanaméricains » et Akan de la Terre Mère en une même communauté transnationale.

Enfin, l'Afro-Américain alors « akanisé » découvrira les divinités vénérées à l'Akonedi shrine. Après avoir reçu un bain spirituel, le disciple se verra remettre des colliers (ou ahene), symbolisant les principales divinités du système akan. Le collier rouge et blanc d'Asuo Gyebi, celui de Nana Esi Ketewaa, bordeaux, blanc et noir, et la chaîne métallique d'Adade Kofi sont en général les premiers qu'il recevra. Parfois, le nouvel adepte obtiendra la bénédiction d'autres divinités ou ancêtres. Il est par exemple assez courant que les représentants américains du clan d'Oparebea, ceux auxquels avait été attribué le patronyme de la prêtresse, lors du rituel du changement de nom, reçoivent rapidement un ahene bleu et blanc représentant Nana Anima, elle-même ancienne prêtresse en chef de l'Akonedi shrine.

Devenu « akan », l'Afro-Américain, membre d'une maison de culte, devra parfaire ses connaissances des divinités et des rituels. Ainsi, il apprendra à faire la 
distinction entre les abosom, divinités qui, à l'Akonedi shrine, sont placées sous la tutelle d'Akonedi, déesse suprême du sanctuaire dont les Ghanéens ont le monopole du culte, les mmoetia, entités spirituelles luttant contre la sorcellerie et caractérisées par leur petite taille, et les nsamanfo, ancêtres du fidèle qui souvent, dans le contexte américain, font figure d'incarnations de ces ancêtres akan réduits en esclavage et déportés dans les plantations du sud des États-Unis. Enfin, s'il est doué de médiumnité et s'il est sujet à l'expérience de la transe, le nouvel adepte sera considéré comme destiné à la prêtrise et devra, tôt ou tard, entamer les trois ans de son périple initiatique. Lors de cette initiation, l'okomfowaa apprendra à exécuter les rituels relatifs à la divinité dont il sera l'officiant, divinité qui sera déterminée, au cours de la première semaine de sa période d'apprentissage, lors d'une séance de reading. Cependant, il ne devra pas pour autant oublier de chercher à favoriser ses relations avec les autres entités, entités avec lesquelles il peut entrer en communication et qu'il peut toutes « posséder » ${ }^{23}$.

Aujourd'hui, les "Akan-américains » entretiennent toujours des rapports étroits avec le Ghana et l'Afrique. Depuis le décès d'Akua Oparebea, en 1995, l'Akonedi shrine est la scène de nombreuses luttes internes relatives à son leadership. À travers ces luttes, la position des Afro-Américains dans le culte est constamment renégociée à la fois par les officiants ghanéens et par le «sacerdoce » américain. La nomination de deux Afro-Américaines à des charges liturgiques de grand prestige - Nana Korantemaa Ayeboafo de Philadelphie est aujourd'hui okomfohene des Akan d'Amérique alors que Nana Attakora de Washington a reçu le titre de reine mère, ohemma, du sanctuaire de la divinité Asuo Gyebi à Larteh Kubease - est largement contestée par une grande majorité des initiés américains et par certains Ghanéens. En réaction à ces nombreux conflits, certains adeptes américains n'hésitent pas aujourd'hui à se forger des liens avec d'autres lieux de culte ghanéens, cherchant notamment à découvrir de nouvelles divinités et de nouveaux rituels. Ce désir; constant chez les fidèles américains, de partir à la quête de nouvelles divinités souvent originaires du nord du Ghana, voire d'autres pays africains, se retrouve dans la série d'alliances que les «Akan-américains » se constituent, aux États-Unis, avec les membres du mouvement yoruba. Introduisant dans leurs croyances, pratiques et rituels des divinités extérieures au monde akan, ils se font les acteurs d'un «syncrétisme panafricain $»{ }^{24}$.

\section{VERS UN SYNCRÉtISME PANAFRICAIN}

Introduite aux États-Unis à la fin des années 1960, « re-territorialisée » dans le Nouveau Monde, la religion akan telle qu'elle est pratiquée à l'Akonedi shrine s'intégre dans un champ religieux afro-américain complexe où elle doit se définir vis-à-vis de cultes populaires à cette époque, la Nation of Islam, ou d'apparition 
récente, le mouvement yoruba. En fait, la réafricanisation au sein de la communauté afro-américaine, née du nationalisme culturel, s'articule comme une réponse aux idéologies de la Nation of Islam et à sa revendication d'une origine musulmane et afro-asiatique de l'homme noir ${ }^{25}$. En effet, pour les leaders de la Nation of Islam, la valorisation de l'ancestralité africaine des Noirs américains constituait un écueil. Aussi insistaient-ils sur l'appartenance de leurs adeptes à une " race » ni africaine, ni américaine, mais qu'ils qualifiaient d'afro-asiatique. Les membres du mouvement, qui adoptaient cette nouvelle identité, se détachaient ainsi des méandres de la « double conscience » évoquée par Du Bois ${ }^{26}$ pour se reconnaître dans une culture et une identité mythique, jugées divines et originelles. Pour les tenants du nationalisme culturel et de la réafricanisation, il s'agira d'invalider l'islam noir et ses revendications afro-asiatiques pour remettre l'Afrique, aussi mystifiée qu'elle soit, au centre des préoccupations noires américaines.

Dans les années 1960, le paysage politico-religieux afro-américain est ainsi déchiré entre des adeptes de la Nation of Islam qui cherchent leur salut dans un islam adapté à leur condition d'homme noir et des fidèles des mouvements yoruba ou akan qui ont fait de la revendication de leur africanité le fondement de leurs pratiques religieuses et de leurs activités politiques. Fondés par des hommes qui, depuis les années 1950, entretiennent d'étroites relations - Dinizulu et Oseijeman Adefunmi, créateur du mouvement yoruba, avaient déjà institué ensemble, en 1955, un premier ordre religieux « africain », l'ordre de Damballah Hwedo qui alliait pratiques yoruba, akan et vadouisantes -, les mouvements yoruba et akan constituent donc un pôle " africain » se déployant à l'encontre de l'islam noir.

Aujourd'hui, il est d'ailleurs intéressant de noter que certains leaders de ces mouvements de réafricanisation participaient eux-mêmes régulièrement, au début de leur carrière religieuse, aux activités de la Nation of Islam. Dans les années 1950 et 1960, la Nation of Islam, alors au faîte de sa gloire, avait attiré de nombreux Afro-Américains intrigués par la revendication d'une origine supérieure de l'homme noir, prônée dans les discours de Malcolm X ou d'Elijah Muhammad, tous deux porte-parole du mouvement. Ainsi, une de mes principales informatrices au sein du mouvement akan a successivement intégré, au cours de son existence, une Église baptiste de la région de Philadelphie (dont sa mère était membre), la Nation of Islam lorsqu'elle est entrée à l'université, un groupe d'islam sunni, le mouvement kemet - organisation prônant le retour des AfroAméricains aux croyances de l'antiquité égyptienne -, avant de devenir une fidèle de la religion yoruba et, enfin, une prêtresse akan. Se pliant aux rituels de changement de nom que pratiquent à la fois la Nation of Islam et le mouvement akan, elle a vu son état civil se transformer au moins à trois reprises passant de Cybil Fequa, son nom chrétien, à Anessah Rasheed, lorsqu'elle intègre la Nation, pour devenir enfin Nana Baakan Agyiriwah. Nana Baakan analyse aujourd'hui son parcours spirituel, du baptisme à la religion akan, comme une évolution 
logique lui ayant permis de mieux comprendre ses origines et de trouver sa place en tant que femme de "descendance africaine » aux États-Unis. Mais si elle a renoncé depuis bien longtemps à la pratique de l'islam, elle considère ses participations aux mouvements yoruba et akan comme un tout, une seule et unique phase de son existence. Dans sa maison de culte sont disposés à la fois des autels akan et yoruba ; c'est avec ces deux types d'entités qu'elle communique régulièrement lors des rituels appelés readings.

L'exemple de Nana Baakan est un cas habituel au sein du champ religieux afro-américain actuel. J'ai, au cours de mes recherches aux États-Unis, rencontré à maintes reprises des prêtres initiés dans les deux systèmes (yoruba et akan), des fidèles "yoruba-américains » s'intéressant aux pratiques ghanéennes et des " Akan-américains » préparant leur initiation à la religion des orisha. En dépit de nombreuses catégories d'accusation entre les deux mouvements ${ }^{27}$, il ne semble pas qu'il y ait, pour ces adeptes-là, de logique de progression entre leur participation à des cérémonies yoruba et leur initiation à la religion akan. Là où, à Cuba, le palo monte ${ }^{28}$ était considéré comme une religion de laquelle l'adepte devait « se rayer » avant de s'initier à la santería, les religions yoruba et akan coexistent sur un pied d'égalité et c'est dans leur association, dans leur cumul qu'elles révèlent leur plus grande efficacité. Ainsi, la maison de culte dirigée par Nana Baakan à Philadelphie accueille régulièrement deux femmes de Baltimore, toutes deux initiées à la prêtrise yoruba au Nigeria. Celles-ci participent aux rituels et sont présentes lors de tous les événements orchestrés par Nana Baakan. Les autres membres de la maison de culte les dénomment tantôt par leurs appellations yoruba, Sangotola et Ogunfumelayo, tantôt par leurs noms akan, Akua et Ama. Les deux femmes ont reçu en 2004 leurs colliers rituels (ahene) et les arborent, lors des cérémonies, mêlés à leurs éléké ${ }^{29}$ yoruba. Pour ces deux adeptes, la participation aux cultes yoruba et akan procède d'une même logique. Pensant être guidées par leurs ancêtres, elles suivent ce qu'elles appellent la « voix de l'Afrique » dans une recherche spirituelle des origines.

En quête permanente de connaissances pouvant prouver leur africanité, les Afro-Américains, adeptes des deux religions, instaurent toute une série d'équivalences entre les deux systèmes, équivalences se manifestant aussi bien dans le rituel que dans la caractérisation des entités spirituelles. La religion akan, par exemple, telle qu'elle est pratiquée aux États-Unis, repose sur la vénération des abosom, abosom qui doivent recevoir trois jours par semaine (le mardi, le vendredi et le dimanche) des offrandes de la part d'adeptes qui, ces jours-là ou shrine days, versent des libations sur leurs autels. « Akanisé », le rituel utilisé par les adeptes des deux systèmes lorsqu'ils communiquent avec leurs orisha suivra alors bien souvent cette logique akan du shrine day. Honorés, selon le calendrier rituel akan, les orisha recevront des libations de gin Gordon, boisson préférée des abosom, accompagnées de prières prononcées dans un mélange d'anglais, de yoruba et de twi (Figure 2). Mais ce sont surtout les divinités elles-mêmes qui, dans un vaste 


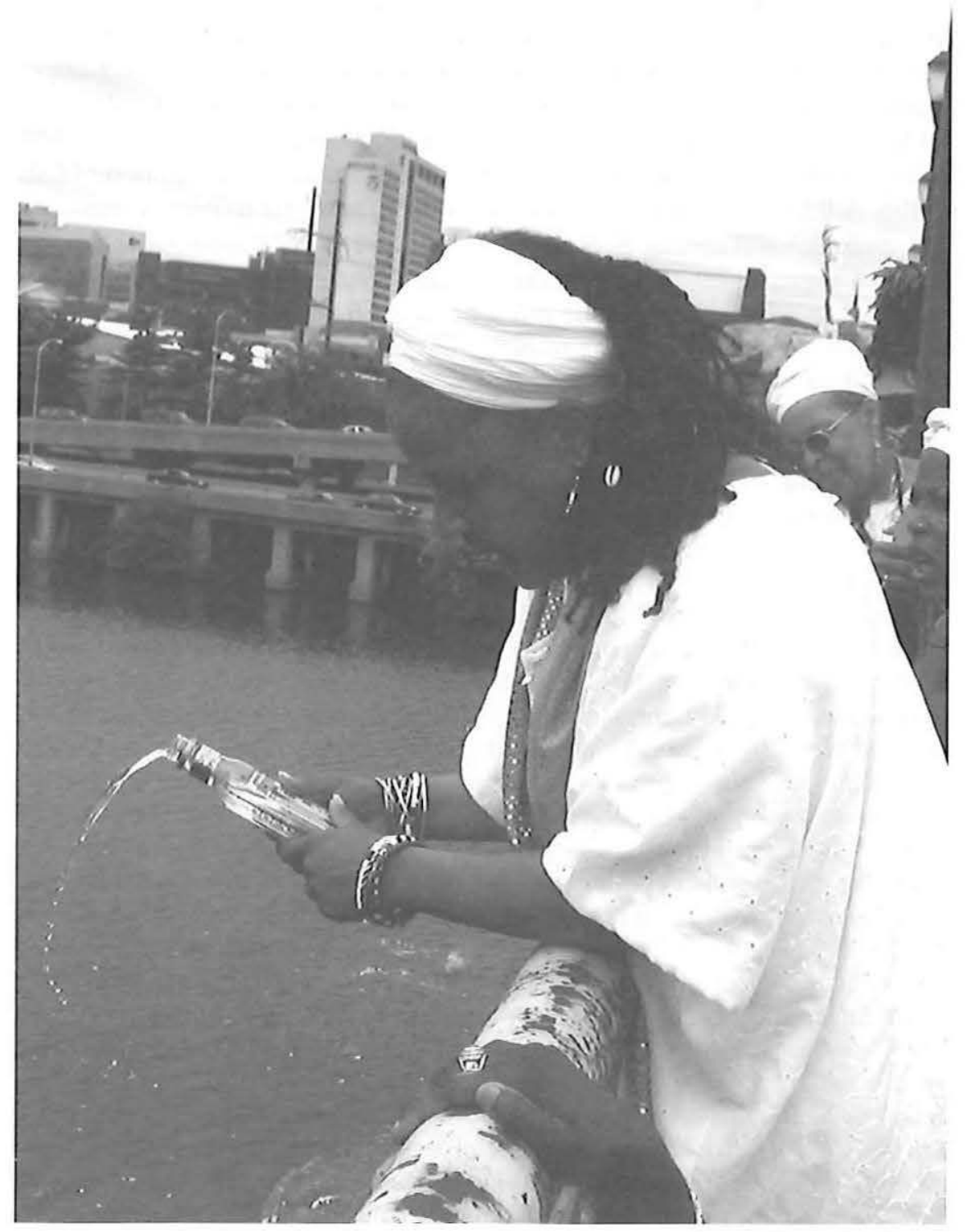

FIg. 2 - À Philadelphie, Nana Baakan Agyiriwah verse des libations de gin en l'honneur de la divinité yoruba Oshun. 
système de croyance unifié, sont associées et confrontées. Si les « abosom et les orisha travaillent ensemble », comme on me le répète si souvent sur le terrain, c'est parce que chaque orisha trouve son équivalent ou son complément dans le monde akan. Ainsi, Asuo Gyebi, l'une des principales divinités akan, est souvent associé au Shangó yoruba, partageant avec celui-ci son arrogance et sa foudre, alors qu'Ogun devient la version yoruba d'Adade Kofi, dieu du fer. De son côté, Tegare, divinité originaire du nord du Ghana, est associé à la figure de l'Eshu yoruba. À l'Akonedi shrine de Larteh Kubease, Tegare est reconnu comme l'un des «enfants » d'Akonedi. Spécialiste de la lutte contre la sorcellerie, il aide la divinité à prescrire les remèdes contrecarrant le $j u j u{ }^{30}$. Mais, aujourd'hui, aux États-Unis, c'est ce même Tegare qui sera considéré par les pratiquants américains comme un messager entre l'ensemble des abosom et le monde des vivants. Ce Tegare, alors " eshuisé », entretient avec son partenaire yoruba d'étroites relations. Souvent représentés par un autel commun, situé à l'entrée de la maison du fidèle, les deux tricksters $^{31}$, Tegare et Eshu reçoivent leurs offrandes ensemble. Nombreux sont les " Akan-américains » qui sont à même de relater certaines des conversations qu'entretiennent Eshu et Tegare lorsque l'adepte se penche sur leur autel ou y verse des libations. Les membres du mouvement qui usent de leur don de medium pour communiquer avec les entités seraient les témoins des relations intimes qui unissent une divinité yoruba à son « homologue » akan.

En fait, tout se passe ici comme si les fidèles des mouvements akan et yoruba cherchaient à forger aux États-Unis un nouveau syncrétisme, syncrétisme panafricain cette fois. Si la réafricanisation s'était fondée aux États-Unis sur la lutte contre le syncrétisme à l'intérieur de la santería cubaine, soit dans un effort de " purification » de la santería de ses influences catholiques, soit dans la formation d'une autre tradition religieuse puisant sa source directement en Afrique, elle traverse aujourd'hui une nouvelle phase qui suppose justement une « resyncrétisation » entre des systèmes de croyances jugés comme authentiquement africains. Là où les esclaves de Cuba auraient dissimulé leurs divinités africaines derrière des saints catholiques ${ }^{32}$, les membres des mouvements yoruba et akan associent entre elles des entités spirituelles africaines. Ce faisant, ils deviennent les détenteurs d'une tradition « africaine » proprement nord-américaine qui les différencie à la fois des pratiquants de la santería et des Africains eux-mêmes, Africains qu'ils jugent corrompus par des siècles de colonialisme. En révélant au grand jour ce qu'ils considèrent comme des équivalences entre deux religions d'Afrique de l'Ouest et en incorporant ces associations dans leurs pratiques et rituels, ils revendiquent, haut et fort, l'existence d'un système de croyances unique, héritage d'une Afrique culturellement unie, oubliée, détruite par les colons européens, mais aujourd'hui «re-territorialisée » aux États-Unis ${ }^{33}$.

Au cœur de cette tentative d'élaboration d'un syncrétisme panafricain ou « panafricanisme rituel " ${ }^{34}$, se trouve l'une des tensions majeures observable au sein de l'expérience de réafricanisation de la communauté afro-américaine. Entre 
création d'une communauté akan ou yoruba transnationale, dont l'AfroAméricain se considère comme le représentant légitime, et formation en Amérique d'un nouveau syncrétisme prônant l'unité d'une Terre Mère «reterritorialisée » aux États-Unis, les négociations entre ethnicité et conscience d'appartenance à une entité panafricaine sont au cœur de la redéfinition de l'identité noire américaine. Se revendiquant les représentants de communautés transnationales qui les unissent à leurs « homologues » africains, en même temps que les uniques détenteurs d'une tradition africaine « ancestrale » et identique à celle de leurs ancêtres avant l'arrivée des Blancs sur la Terre Mère, les AfroAméricains, membres des mouvements yoruba et akan, se font les acteurs d'une histoire nationaliste noire américaine qui, de Marcus Garvey à Louis Farrakhan ${ }^{35}$, a toujours oscillé entre rêves panafricanistes et revendications d'un particularisme afro-américain, d'une exception noire nord-américaine. Se considérant comme les garants d'une tradition originelle, qu'ils pensent avoir la mission divine de raviver, et reconnus par leurs "pairs » africains, qui les ont initiés dans leurs systèmes de croyances, les Afro-Américains des États-Unis se présentent comme les derniers Africains « authentiques», les seuls dont l'« essence » n'aurait pas souffert des tragiques abus du régime colonial et aurait survécu indemne aux horreurs de l'esclavage.

\section{Conclusion}

Depuis les années 1970, la réafricanisation de la communauté afroaméricaine aux États-Unis est ainsi sans cesse redéfinie par les relations entretenues entre deux de ses principaux représentants : les mouvements yoruba et akan. Réafricanisation " akanoyoruba - centrée », elle est le produit des allées et venues constantes de certains de ses adeptes entre deux mouvements qui ont, dès leurs origines, coexisté et collaboré. Fondés tous deux sur une tentative de créer des communautés transnationales se déployant entre le Nouveau Monde et la Terre Mère, les mouvements yoruba et akan ont donné naissance, sur le territoire américain, à un nouveau syncrétisme sur lequel plane l'héritage des décennies de luttes panafricaines.

Toutefois, plusieurs tenants de la réafricanisation défendent aujourd'hui une position différente qui va à l'encontre de l'élaboration d'un syncrétisme panafricain. Ainsi, Kimati Dinizulu, fils de Nana Yao Opare, accuse certains « Akanaméricains » de nier ce qu’il considère comme « la véritable tradition akan ", tradition dont son père fut le premier représentant en Amérique. Dans une même logique, John Mason, babalorisha ${ }^{36}$ afro-américain et auteur de plusieurs ouvrages sur les rituels yoruba, estime, pour sa part, que ces associations entre systèmes yoruba et akan sont des créations nord-américaines qui ne font qu'éloigner les Afro-Américains de leurs « réelles » traditions ancestrales. Ainsi, aujourd’hui, un 
nouveau débat divise les tenants de la réafricanisation aux États-Unis. Opposant les partisans du syncrétisme panafricain aux fidèles des traditions akan et yoruba, lesquelles se voudraient originaires de régions précises en Afrique et transposées dans le Nouveau Monde, ce débat prend la forme d'une lutte autour de l'acception de cette tradition africaine que les Afro-Américains, adeptes des théories du nationalisme culturel, se donnent pour mission de raviver dans l'espoir de vivre un jour librement en Amérique, conformément à leur présumé héritage culturel. *

* Manuscrit reçu en janvier 2005, accepté pour publication en juin 2005.

\section{NOTES}

1. Sur la réafricanisation au Brésil, voir Capone (1999a).

2. Les odìs sont les combinaisons obtenues au cours d'une divination liée au système d' lfá, système maîtrisé par des spécialistes appelés à Cuba babalaos.

3. Sur les tentatives de réafricanisation de la santería à Cuba, voir Argyriadis et Capone (2004).

4. Sur l'utilisation de la théorie des masques de Bastide dans la réafricanisation, voir Capone (1999a).

5. Voir Capone (1999a ; 2000a).

6. Les États-Unis, l'Argentine ou le Mexique constituent ce que Alejandro Frigerio (2004) désigne sous l'appellation de " diasporas religieuses secondaires ». Ces foyers plus ou moins récents ont adopté des pratiques religieuses, comme la santería ou le candomblé, développées dans des foyers de « diaspora primaire » tels Salvador de Bahia ou La Havane. Sur la réafricanisation en Argentine, voir Frigerio (1989) ou Oro (1994). Sur la pratique de la santeria au Mexique, voir Juárez Huet (2004). C'est de la réafricanisation aux États-Unis dont il sera question dans cet article. Pour un tableau de cette expérience en Amérique du Nord, voir également Capone (1999b ; s.d.), Guedj $(2003 ; 2004)$ et Palmié (1995).

7. Sur le nationalisme culturel aux États-Unis, voir Guedj (2004).

8. L'identité yoruba revendiquée par les Afro-Américains est le produit d'une construction identitaire et non l'expression d'une origine ethnique réelle. Afin de bien différencier ces « Yoruba de la diaspora " des Africains, j'utiliserai ici le terme de "Yoruba-américains " pour désigner les Noirs d'Amérique du Nord pratiquants de la religion des orisha. Dans une même logique, les membres du mouvement akan seront qualifiés d'« Akan-américains ».

9. Les " Akan-américains » utilisent à la fois des termes twi et anglais pour décrire leurs croyances et leurs pratiques. Plutôt que de chercher à uniformiser les termes dans une langue unique, j'ai respecté les choix des acteurs eux-mêmes lorsqu'ils passent d'une langue à l'autre.

10. Sur les notions de communauté et de réseaux transnationaux, voir, entre autres, Clifford (1997) et Hannertz (1996). Pour une réflexion sur l'apport des études afro-américaines dans une tentative de conceptualisation de ces phénomènes, voir Capone (2004)

11. Sur la constitution d'identités ethniques transnationales akan et yoruba, voir Capone (2000b) et Guedj (2004). Dans ce dernier texte, paru dans la revue Civilisations, j’ai analysé comment la constitution de communautés akan et yoruba se déployant entre l'Afrique et l'Amérique avait abouti à la formation d'un nouveau nationalisme afro-américain, un nationalisme transnational, qui n'appuie plus ses revendications sur l'existence d'une Nation noire ancrée à l'intérieur du territoire américain, mais sur la création de groupes ethniques dé-territorialisés.

12. Sur les relations entretenues entre le Ghana et les États-Unis, voir Laronce (2000).

13. Sur Marcus Garvey, voir Cronon (1955). Sur l'influence de Marcus Garvey sur le discours nationaliste afro-américain actuel, voir Guedj (2004). 
14. Sur la conception du panafricanisme de Du Bois, voir, entre autres, Marable (1999).

15. Aujourd'hui, la maison de Du Bois à Accra abrite une bibliothèque spécialisée sur le panafricanisme et est le chef-lieu de l'African American Association of Ghana, association rassemblant des Afro-Américains qui, comme Du Bois, sont venus s'installer dans le pays. La visite guidée de cet édifice comporte un passage par le bureau de Du Bois où il commença la rédaction de l'encyclopédie qu'il ne put achever et un recueillement sur sa tombe située dans un mausolée non loin de son habitation.

16. Je tiens ici à remercier le programme de l'IFRA, Réseaux transnationaux et nouveaux acteurs religieux en Afrique de l'Ouest, qui m'a permis en janvier et février 2003 d'effectuer une première enquête de terrain à l'Akonedi shrine de Larteh Kubease au Ghana.

17. Voir à ce sujet, Mensah Dapaa (1961).

18. Sur la Ghana psychic and traditional healing association, voir Evans-Anfom (1986).

19. Sur les efforts de Nkrumah pour tenter de créer un Ghana uni, voir son livre publié en 1959.

20. Il existe aujourd'hui de rares maisons de culte qui ne revendiquent pas leur filiation avec l'Akonedi shrine. Les officiants de ces maisons ont été initiés dans d'autres sanctuaires ghanéens, souvent dans la région ashanti ou dans le nord du pays.

21. Les "Akan-américains " appellent readings les rituels durant lesquels l'okomfo échange des informations avec les entités spirituelles après avoir versé des libations sur leurs autels.

22. La pratique du changement de nom se retrouve dans la plupart des mouvements nationalistes afro-américains, du Moorish Science Temple musulman aux mouvements akan ou yoruba. Pour une réflexion sur le changement de nom au sein de la Nation of Islam, mouvement noir le plus populaire aux États-Unis jusqu'au début des années 1990, voir, entre autres, Gardell (1996) et Guedj (2000).

23. Il est intéressant de noter que les "Akan-américains 》 ont tendance à se décrire comme les agents de la possession par les divinités. Ce ne serait donc pas, dans cette logique, les entités qui possèderaient les pratiquants, mais les fidèles qui prendraient la décision d'entrer en transe, de posséder les abosom, nsamanfo ou mmoetia.

24. Au Ghana, l'Akonedi shrine, sanctuaire situé dans une ville guan, rassemble des divinités originaires de différentes régions de ce pays. En effet, Tegare et Asuo Gyebi sont originaires du Nord alors que Nana Esi Ketewaa est fanti. Pour certains initiés américains, cette union de divinités sous l'appellation d'«akan » constitue une première expérience de "panafricanisme religieux » ou " rituel ", mise en cuvre déjà sur le territoire ghanéen. Sur la notion de panafricanisme rituel, voir Capone (s.d.).

25. Sur les relations entre islam afro-américain et réafricanisation, ainsi que pour une réflexion autour de la notion d'identité afro-asiatique, voir Guedj (2003).

26. Dans son ouvrage, The Souls of Black Folk (1994 [1903]), Du Bois évoque, pour la première fois, le thème de la " double conscience " sur lequel il reviendra à maintes reprises au cours de sa carrière. Par "double conscience ", cet auteur entend ce malaise qui conditionne, selon lui, la place des AfroAméricains dans la société américaine. À la fois Noirs aux origines africaines, appartenant à une minorité méprisée et citoyens américains, les Afro-Américains des États-Unis ne pourront améliorer leur condition qu'en cherchant des solutions au dilemme posé par cette double appartenance.

27. La principale catégorie d'accusation, utilisée à la fois par les initiés "yoruba-américains » à l'encontre des " Akan-américains » et par les membres du mouvement akan à l'encontre des « Yorubaaméricains ", concerne la possession. Alors que les "Yoruba-américains " critiquent souvent la capacité des " Akan-américains » à se faire posséder, lors des cérémonies, par l'ensemble des entités de leur système ainsi que par les orisha, les « Akan-américains » ont tendance à retourner cette accusation en décrivant les pratiquants de la religion des orisha comme de piètres spécialistes de la transe qui ne savent maîtriser que les possessions provoquées par l'entité dont ils sont les ofliciants.

28. Le terme palo monte désigne une modalité de culte afro-cubaine qui se concentre autour de la vénération d'esprits et de divinités reconnues comme d'origine bantou.

29. Le terme éléké désigne les colliers rituels remis à l'adepte de la religion yoruba au début de son périple initiatique. 
30. Juju et black magic sont les termes généralement utilisés par les « Akan-américains » et par les Ghanéens de l'Akonedi shrine pour désigner les actes de sorcellerie.

31. Généralement utilisé pour décrire le comportement d'Eshu, ce qualificatif s'applique aujourd'hui également à la personnalité de Tegare.

32. Voir note 5 .

33. La revendication de l'existence d'une Afrique unie et détruite par le colonialisme est un cheval de bataille important pour l'historien sénégalais Cheikh Anta Diop. Pour une énonciation de cet argument, voir Diop (1959). Pour une analyse de l'influence des écrits de Cheikh Anta Diop sur le nationalisme afro-américain, voir Guedj (2003).

34. La notion de panafricanisme rituel est utilisée par Stefania Capone (s.d.) dans son analyse du mouvement yoruba.

35. Louis Farrakhan est l'actuel leader de la Nation of Islam. À l'image de son prédécesseur, the Honorable Elijah Muhammad, il considère les membres de sa Nation comme des Original Men, descendants afro-américains d'Allah, qui ont aujourd'hui la mission de sauver l'ensemble des populations noires de la planète. Voir, à ce sujet, le site Internet de la Nation of Islam, http://www.noi.org, qui rassemble les principaux textes rédigés par les porte-parole du mouvement.

36. Un babalorisha est le chef d'une maison de culte de santería, appelé aussi padrino. Voir, entre autres, Mason (1993).

\section{BIBLIOGRAPHIE}

\section{Argyriadis Kali et Stefania CAPONE}

2004 "Cubania et Santería. Les enjeux politiques de la transnationalisation religieuse (La Havane-Miami) », Civilisations, LI (1-2), pp. 81-137.

CAPONE Stefania

1999a La quête de l'Afrique dans le candomblé. Pouvoir et tradition au Brésil, Karthala, Paris.

1999b «Les dieux sur le Net : l'essor des religions d'origine africaine aux ÉtatsUnis ", L'Homme, 151, pp. 47-74.

2000a "Entre Yoruba et Bantou: l'influence des stéréotypes raciaux dans les études afro-américaines ", Cahier d'études africaines, 157, pp. 55-77.

$2000 \mathrm{~b}$ "D'une identité religieuse à une identité "ethnique" : la communauté transnationale des pratiquants de la religion des orisha ", communication présentée au VIII Congreso Latinoamericano de religión y etnicidad, Padoue, Italie, 30 juin-5 juillet.

2004 "A propos des notions de globalisation et de transnationalisation », Civilisations, LI (1-2), pp. 9-22.

s.d. Les Yoruba du Nouveau Monde. Religion, ethmicité et nationalisme noir aux États-Unis.

\section{CLIFFORd James}

1997 Routes. Travel and Translation in the Late Twentieth Century, Harvard University Press, Cambridge, Mass., Londres.

Cronon Edmund

1955 Black Moses. The story of Marcus Garvey and the universal Negro improvement association, University of Wisconsin Press, Madison. 
Diop Cheikh Anta

1959 L'unité culturelle de l'A frique noire, Présence africaine, Paris.

Du BoIs William Edward Burghandt

1994 The Souls of Black Folk, Random House, New York [1903].

Evans-ANFom Eric

1986 Traditional medicine in Ghana : practices, problems and prospects, J. B. Danquah Memorial Lectures, Accra.

Frigerio Alejandro

1989 With the Banner of Oxala. Social construction and maintenance of reality in Afro-Brazilian religions in Argentina, Ph.D Dissertation, Anthropology Dept, University of California, Los Angeles.

2004 "Reafricanization in secondary religious diasporas : constructing a World Religion », Civilisations, LI (1-2), pp. 39-60.

Gardell. Mattias

1996 In the name of Elijah Muhammad : Louis Farrakhan and the Nation of Islam, Duke University Press, Durham.

GuEds Pauline

2000 La Nation de l'Islam aux États-Unis: de l'éthique de pureté de la race à l'idéologie séparatiste, mémoire de maîtrise, université Paris X-Nanterre, Nanterre.

2003 "Des "Afro-Asiatiques" et des "Africains" : islam et afrocentrisme aux États-Unis ", Cahiers d'études africaines, XLIII (4), 172, pp. 739-760.

2004 "A Nation within Nations" : nationalisme afro-américain et réafricanisation aux États-Unis ", Civilisations, LI (1-2), pp. 23-38.

HANNERTZ UIf

1996 Transnational Connections, Routledge, Londres/New York.

JuÁrez HuEt Nahayeilli B.

2004 «La santería dans la ville de Mexico : ébauche ethnographique », Civilisations, LI (1-2), pp. 61-79.

Laronce Cécile

$2000 \quad$ Nkrumah, le panafricanisme et les États-Unis, Karthala, Paris.

MARABLE Manning

1999 "The Pan-Africanism of W.E.B. Du Bois », in Black Leadership, Penguin Books, New York, pp. 75-96.

MASON John

1993 Four New World Yorùbá Rituals, Yorùbá Theological Archministry, Brooklyn, N.Y. [1985].

MENSAH DAPAa W. S.

1961 "Observations on Traditional Healing Methods in Ghana », Ghana Journal of Science, 1 (1-2), pp. 523-536.

NKrumah Kwame

1959 Ghana : the autobiography of Kwame Nkrumah, Thomas Nelson and Sons Ltd., Edimbourg.

1968 The Spectre of Black Power, Panaf Books, Londres. 
JOURNAL DE LA SOCIÉTÉ DES AMÉRICANISTES

Oro Ari Pedro

1994 Axé Mercosul. As religiōes afro-brasileiras nos paises do Prata, Vozes, Petrópolis.

Palmié Stephan

1995 "Against syncretism: "Africanizing" and "Cubanizing". Discourses in North American Orisà Worship ", in R. Fardon, éd., Counterworks : managing the diversity of knowledge, Routledge, Londres, pp. 73-104. 